\title{
Complicaciones de la cirugía craneofacial en tumores de base craneal anterior
}

\author{
A. Torres; J.J. Acebes; L. López; A. Marnov; J. Viñals*; J.M. Serra*; M. Maños** y A. Monner***
}

Servicios de Neurocirugía, Cirugía Plástica*, ORL** y Cirugía Maxilofacial***. Hospital Universitario de Bellvitge. Barcelona.

Resumen

Introducción. Los recientes avances en radiología, técnica microquirúrgica y de reconstrucción han permitido que la cirugía craneofacial (CCF) se haya convertido en el tratamiento de elección de la patología tumoral de fosa craneal anterior. A pesar de esto, continua asociándose a una incidencia de complicaciones considerable (24-56\%).

Objetivos. Revisión, análisis y manejo de las complicaciones de la CCF en una serie de 41 pacientes.

Material y métodos. 41 pacientes diagnosticados de patología tumoral infiltrativa de base craneal anterior, fueron intervenidos mediante CCF, entre 1990 y 2002. La extensión tumoral fue valorada en todos los casos con TC y RMN de la región craneo-facial. Tras determinar el volumen y extensión tumoral se diseñó un tratamiento quirúrgico radical, con el objetivo de conseguir una resección tumoral "en bloque".

Resultados. El $63.4 \%$ de los pacientes eran varones con una mediana de edad de 57 años (18-73). La histología más frecuente de los tumores fue carcinoma escamoso y la localización habitual fue en los senos paranasales en el $78 \%$ ( 32 de 41 casos). En el $85.4 \%$ de los pacientes se realizó una craneotomía bifrontal basal $y$ en el $14.6 \%$ restante craneotomía frontoorbitaria unilateral, en un sólo colgajo óseo. El área de resección facial incluía: etmoidectomía (60.9\%), etmoido-esfenoidectomía $(24.3 \%)$, maxilectomía unilateral $(39 \%)$ y exenteración orbitaria (14.6\%). Para la reconstrucción se utilizó: colgajo pediculado pericraneal $(\mathbf{1 0 0 \%})$, colgajos locales $(34.1 \%)$, colgajos libres microvascularizados (21.9\%) e injerto óseo autólogo (19.5\%). 20 pacientes $(48.7 \%)$ presentaron complicaciones postoperatorias, entre las que destacan, como complicaciones mayores la fístula de LCR $(\mathbf{1 2 . 1 \% )}$ y la meningitis (7.3\%). La mortalidad global fue del $7.3 \%$.

Conclusiones. La CCF continua asociándose a una elevada tasa de complicaciones, en general de tipo

Recibido: 29-09-04. Aceptado: 20-02-05 infeccioso. El perfeccionamiento de las técnicas de reconstrucción permitirán reducir la incidencia de las complicaciones más frecuentes y peligrosas.

PALABRAS CLAVE: Cirugía craneofacial. Base de cráneo. Técnica quirúrgica. Complicaciones.

Complications of craniofacial resection in anterior skull base tumors

Summary

Introduction. The development of new radiographic techniques and the refinement of microsurgery and reconstructive surgery have been the responsible of the establishment of craniofacial resection (CFR) as the standard treatment of anterior skull base tumors. Overall complication rates varies from $24-56 \%$, according to a review of recently published series.

Objectives. To describe the complications of CFR in a series of $\mathbf{4 1}$ patients and to analyze the management and final outcome.

Material and methods. From 1990 to 2002, 41 patients underwent CFR for tumors involving the anterior cranial base. The extent of the tumor was always assessed with craniofacial CT-scan and MRI. The objective of the surgical treatment was to achive "on block" removal of the tumor.

Results. The average age was 57 years with a male preponderance (63.4\%). Squamous cell carcinoma was the most frequent histopathological type of tumor. The tumors were localized in paranasal sinuses in $78 \%$ of the cases. Bifrontal craniotomy was performed in $85.4 \%$ and unilateral orbitofrontal craniotomy in $14.6 \%$ of the cases. The area of facial resection included: ethmoidectomy (60.9\%), ethmoido-sphenoidectomy (24.3\%),

Abreviaturas. CCF: cirugía craneofacial. CFR: craniofacial resection. CSF: cerebrospinal fluid. LCR: líquido cefalorraquídeo. ORL: otorrinolaringología. RDT: radioterapia. RM: resonancia magnética. 
maxillectomy (39\%) and orbital exenteration (14.6\%). The reconstruction of the floor of the anterior cranial fossa was performed using pedicled pericranial flap $(100 \%)$, local $(34.1 \%)$ or microvascular free flaps $(21.9 \%)$ and split calvarian graft (19.5\%). 20 patients (48.7\%) developed post-operative complications, CSF leaks $(12.1 \%)$ and meningitis $(7.3 \%)$ being the most frequent major complications. The mortality rate was $7.3 \%$.

Conclusions. CFR has become the standard approach for anterior cranial base tumors. Despite its widespread application, the complication's rate ranges between $24-50 \%$ and the procedure carries a risk of significant morbidity and even mortality. Improvement of specific aspects of surgical technique and more refined reconstructive methods will decrease the number of complications.

KEY WORDS: Craniofacial resection. Skull base. Surgical technique. Complications.

\section{Introducción}

La cirugía craneofacial (CCF) es actualmente el tratamiento estándar de la patología tumoral de fosa craneal anterior. Utilizada inicialmente por Dandy ${ }^{7}$ en la resección de tumores orbitarios en 1941 y posteriormente por Ketcham $^{15}$ en el tratamiento quirúrgico de tumores de senos paranasales con extensión a la base de cráneo ${ }^{14,15,23,26}$, la CCF ha ido experimentando una gran evolución en los últimos años.

Los recientes avances en radiología, técnica microquirúrgica y reconstrucción, así como el desarrollo de equipos multidisciplinarios han permitido que la CCF se haya convertido en un tratamiento seguro y eficaz en este tipo de patología $a^{9,11,13,21,28}$. A pesar de que la incidencia de complicaciones cada vez es menor, todavía oscila entre el $24-56 \%$ según las series más recientes ${ }^{21,24,28}$, entre las cuales, la fístula de LCR y las infecciones son las más frecuentes y las que comportan mayor morbi-mortalidad.

Incorporando muchos de estos nuevos avances, presentamos nuestra experiencia en CCF con una serie de 41 casos de patología tumoral con invasión de base craneal anterior, intervenidos entre 1990-2002. Hemos prestado especial atención a las complicaciones post-operatorias y al manejo terapéutico de las mismas, con el objetivo de detectar los factores asociados a su aparición y optimizar así el tratamiento quirúrgico.

\section{Material y Métodos}

Un total de 41 pacientes, con diagnóstico de patología tumoral infiltrativa de base craneal anterior, fueron interve- nidos mediante CCF, entre 1990 y 2002.

La extensión tumoral fue valorada en todos los casos con TC y RMN de la región craneo-facial en proyecciones axiales y coronales. Se realizó un estudio oftalmológico preoperatorio a todos los pacientes que presentaban síntomas visuales al diagnóstico.

En el 48.7\% (20 de 41 casos) se realizó biopsia preoperatoria. Tras determinar el volumen y extensión tumoral se diseñó un tratamiento quirúrgico radical (CCF), cuyo objetivo final fue conseguir una resección tumoral "en bloque".

\section{Resección craneofacial}

Los detalles técnicos de la CCF han sido previamente descritos $^{1.2,6,10,16,17,22,23,27,29}$ y son adaptados a cada caso individual y tipo de tumor. La traqueostomía se practicó en el $12.1 \%$ de los pacientes ( 5 de 41 ), todos ellos con extensas lesiones tumorales de senos paranasales y compromiso de la vía aérea, con el objetivo de facilitar el abordaje quirúrgico y evitar complicaciones respiratorias tras la cirugía. Se realizó profilaxis antibiótica en todos los pacientes. No utilizamos el drenaje lumbar tras la inducción anestésica en ningún caso.

El abordaje practicado fue una incisión cutánea bicoronal, preservando el pericráneo, para utilizarlo posteriormente en la reconstrucción del suelo de la fosa craneal anterior. Para prevenir la retracción excesiva de los lóbulos frontales y garantizar una adecuada exposición realizamos una craneotomía bifrontal amplia y basal, incluyendo el seno frontal, el cual fue cranealizado en todos los casos. En los casos en que existía extensión orbitaria (6 casos) se realizó una craneotomía bifronto-orbitaria unilateral en un sólo colgajo óseo.

La resección de la base craneal fue diseñada en función de la localización, tamaño y extensión de la porción tumoral extradural, realizando osteotomías en la lámina cribiforme, planum esfenoidal y techos orbitarios, bajando el fragmento craneal aislado en la craneotomía.

En aquellos casos en que tanto las pruebas de imagen como el aspecto macroscópico no demostró infiltración dural ni del parénquima cerebral (29 de 41), se realizó una resección extradural.

Cuando se objetivó infiltración dural macroscópica (12 de 41 casos), la inspección y resección intradural del tumor se completó antes de la apertura de los senos paranasales, para evitar la contaminación de estructuras del SNC. Durante la exploración del lóbulo frontal se detectó infiltración del parénquima cerebral en 3 de estos 12 casos. Finalizada la inspección/resección intradural, el defecto dural basal se reparó de forma primaria o mediante un injerto autólogo de pericráneo o fascia muscular.

El abordaje transfacial se practicó mediante rinotomía 
TABLA 1

Clasificación histológica de los tumores intervenidos

\begin{tabular}{l|c}
\multicolumn{1}{c|}{ Tipo histológico } & N de casos \\
\hline Carcinoma escamoso & 9 \\
Carcinoma basocelular & 7 \\
Adenocarcinoma & 7 \\
Sarcoma & 5 \\
Carcinoma adenoide quístico & 4 \\
Displasia fibrosa & 2 \\
Papiloma escamoso & 2 \\
Papiloma invertido & 1 \\
Melonoma & 1 \\
Neuroblastoma & 1 \\
Cordoma condroide & 1 \\
Swanoma maligno & 1 \\
Total & 41 \\
\hline
\end{tabular}

lateral o incisión cutánea tipo Weber-Ferguson. La resección tumoral tridimensional "en bloque" fue elaborada siguiendo unos márgenes anatómicos naturales; de esta manera, para tumores etmoidales la resección incorporó la pared lateral y el septum nasal, las celdillas etmoidales y las paredes mediales orbitarias. Cuando la extensión o localización tumoral hizo necesaria una resección más amplia, la resección se amplió a otras estructuras faciales como el maxilar o el esfenoides y en el caso de extensión orbitaria se realizó la exenteración orbitaria. En aquellos casos en que se practicó maxilectomía medial con preservación orbitaria, se reconstruyó el ligamento cantal. Para prevenir estenosis lacrimales y epíforas post-quirúrgicas se introdujeron "stents" en los conductos lacrimales.

\section{Reconstrucción}

En todos los procedimientos, el abordaje craneofacial fue realizado por un equipo multidisciplinar formado por neurocirujanos, cirujanos plásticos, cirujanos maxilofaciales u otorrinolaringólogos.

Tras realizar la CCF, es muy importante una adecuada

Tabla 2

Area de resección cráneo-facial

\begin{tabular}{l|c}
\hline Tipo de resección & $\mathbf{N}^{\circ} \mathbf{d e} \mathbf{c a s o s}(\mathbf{\%})$ \\
\hline Craneotomía bifrontal & $35(85.3 \%)$ \\
Craneotomía bifrontal +orbitotomía unilateral & $6(14.6 \%)$ \\
Etmoidectomía & $25(60.9 \%)$ \\
Etmoido-esfenoidectomía & $10(24.3 \%)$ \\
Maxilectomía unilateral & $16(39 \%)$ \\
Exenteración orbitaria & $6(14.6 \%)$ \\
\hline
\end{tabular}

Tabla 3

Número de complicaciones en relación con existencia de invasión dural tumoral y tipo de reconstrucción

\begin{tabular}{|c|c|c|c|c|}
\hline & & $\begin{array}{l}\text { Fístula LCR } \\
\mathrm{N}^{\circ} \text { casos (\%) }\end{array}$ & $\begin{array}{c}\text { Meningitis } \\
\mathrm{N}^{\circ} \operatorname{casos}(\%)\end{array}$ & $\begin{array}{l}\text { Fallo colgajo } \\
\mathrm{N}^{\circ} \operatorname{casos}(\%)\end{array}$ \\
\hline Invasión dural & $\mathbf{N}^{\circ} \operatorname{casos}(\%)$ & & & \\
\hline Invasión dural + & $12(29.2)$ & $3 / 12(25)$ & 2/12(16.6) & \\
\hline Invasión dural - & $29(70.8)$ & $2 / 29(6.8)$ & $1 / 29(3.4)$ & \\
\hline Reconstrucción & $\mathrm{N}^{\circ} \operatorname{casos}$ & & & \\
\hline Colgajo libre & 9 & & & \\
\hline Recto abdominal & $4 / 9$ & & & $1 / 4(25)$ \\
\hline Fascio-cut. radial & $5 / 9$ & & & $1 / 5(20)$ \\
\hline Colgajo local (M temp.) & 14 & & & $4 / 14(28)$ \\
\hline
\end{tabular}

LCR: líquido cefaloraquídeo. Fascio-cut. radial: fascio-cutáneo de radial. M. temp: músculo temporal 
Tabla 4

Complicaciones post-quirúrgicas

\begin{tabular}{l|lc}
\hline \multicolumn{2}{l}{ Tipos de complicaciones } & No casos (\%) \\
\hline Neurológicas & Hematoma epidural agudo & $4(9.7 \%)$ \\
& Meningitis & $3(7.3 \%)$ \\
\multirow{2}{*}{ Locales } & Neumoencéfalo & $2(4.9 \%)$ \\
& Infección de herida quirúrgica & $10(24.3 \%)$ \\
& Fístula de LCR & $5(12.1 \%)$ \\
& Fallo de colgajo local pediculado & $4(9.7 \%)$ \\
& Diplopia & $3(7.3 \%)$ \\
& Fallo de colgajo libre vascularizado & $2(4.9 \%)$ \\
& Fístula nasocutanea & $2(4.9 \%)$ \\
& Obstrucción nasolacrimal & $1(2.4 \%)$ \\
& Osteomielitis & $1(2.4 \%)$ \\
& Neumonia/Atelectasia & $6(14.6 \%)$ \\
& Shock séptico & $2(4.9 \%)$ \\
& Trombosis venosa profunda & $1(2.4 \%)$ \\
\hline
\end{tabular}

reconstrucción de los grandes defectos craneofaciales de huesos y tejidos blandos. En aquellos casos en que existía infiltración tumoral dural, realizamos sellado dural hermético (con o sin injerto). Para la reparación del defecto de la fosa craneal anterior, restableciendo la barrera entre la dura frontobasal y los senos paranasales, cavidad nasal y nasofaringe utilizamos el colgajo pericraneal pediculado en todos los casos. Cuando el defecto de la base craneal fue superior a $5 \mathrm{~cm}$ en cualquiera de sus diámetros, se reconstruyó con injerto óseo autólogo de diploe craneal, dando así soporte y suspensión a las estructuras neurales.

Otro factor clave para evitar el desarrollo de complicaciones es evitar los espacios muertos, para ello realizamos la transposición muscular local con colgajo de músculo temporal, o colgajo muscular libre microvascularizado (recto abdominal o colgajo libre fascio-cutáneo de radial).

Hemos analizado detalladamente nuestra técnica quirúrgica, prestando especial atención en la aparición de complicaciones post-operatorias y su manejo, así como la mortalidad quirúrgica.

\section{Resultados}

Entre 1990 y 2002, 41 pacientes fueron intervenidos mediante CCF. El $63.4 \%$ de los pacientes eran varones con una mediana de edad de 57 anos (18-73). La histología más frecuente de los tumores fue carcinoma escamoso (Tabla 1) y el origen tumoral fue los senos paranasales en el $78 \%$ ( 32 de 41 casos) y la órbita en el $14.6 \%$ (6 casos). El 24.3\% (10 de 41) de los pacientes habían recibido algún tipo de tratamiento previo y presentaban recidiva/ persistencia tumoral. Todos los pacientes de este subgrupo habían sido intervenidos con anterioridad, la mitad (5 de 10) habían recibido radioterapia y 2 pacientes radioterapia y quimioterapia.

\section{Resección craneo-facial}

El abordaje craneal consistió en una craneotomía bifrontal basal incluyendo el seno frontal en el $85.4 \%$ de los casos. En los casos en que era necesario practicar una exanteración orbitaria se realizó craneotomía frontoorbitaria unilateral en un sólo colgajo óseo, 14.6\% (Tabla 2).

Se identificó infiltración dural macroscópica en el $29.2 \%$ de los casos (12 de 41) y afectación del parénquima cerebral en el 7.3\% (3 de 41). Dentro del subgrupo de pacientes que presentaban afectación dural, el $25 \%$ ( 3 de 12 pacientes) y el $16.6 \%$ ( 2 de 12 ) presentaron fístula de LCR y meningitis post-quirúrgica respectivamente.

El área de resección facial fue el siguiente: etmoidectomía en el $60.9 \%$ de los casos, etmoido-esfenoidectomía en el $24.3 \%$, maxilectomía unilateral en el 39\% y exenteración orbitaria en el 14.6\% (Tabla 2).

\section{Reconstrucción}

El colgajo pediculado pericraneal se utilizó en el 100\% de los casos para la reconstrucción de la base craneal, a lo que se añadió, en los defectos superiores a $5 \mathrm{~cm}$, injerto 
óseo autólogo de diploe craneal (8 casos). Se diseñaron 14 colgajos locales de músculo temporal pediculado y 9 colgajos libres microvascularizados (anastomosados a vasos cervicales): 4 colgajos de músculo recto abdominal y 5 colgajos fascio-cutáneos antebraquiales radiales (Tabla 3).

\section{Complicaciones post-quirúrgicas}

Un total de 20 pacientes (48.7\%) desarrollaron complicaciones post-operatorias, de las cuales el $78.1 \%$ eran potencialmente graves (36 de 46). Hemos clasificado las complicaciones en neurológicas, locales y sistémicas (Tabla 4). La complicación neurológica más frecuente $(9.7 \%)$ fue el hematoma epidural agudo (siendo necesario en un caso la evacuación quirúrgica). El 7.3\% de los pacientes desarrollaron meningitis ( 3 casos), todos ellos tras fístula de LCR previa. Dentro del conjunto de complicaciones locales, destacan las infecciones superficiales de herida quirúrgica en 10 casos. La incidencia global de fístula de LCR fue del $12.1 \%$, aunque en el subgrupo de pacientes en que existía infiltración tumoral dural aumentó hasta el 25\% (Tabla 3). Los fallos de colgajo libre microvascularizado y local pediculado aparecieron 2 veces en el primero y 4 en el segundo, requiriendo la revisión quirúrgica en todos ellos. La complicación sistémica más frecuente fue la infección respiratoria en 6 casos y la complicación asociada a mayor mortalidad fue el shock séptico ( 2 casos).

La incidencia global de mortalidad post-quirúrgica fue del 7.3\% (3 casos), dos de ellos tras shock séptico y uno tras meningitis.

\section{Discusión}

La CCF ha experimentado en los últimos años grandes cambios, asociados a la aparición de nuevas técnicas radiológicas, al desarrollo de la microcirugía y de las técnicas de reconstrucción. Esto ha permitido que la CCF se haya convertido en la actualidad en el tratamiento de elección de la patología tumoral (tanto benigna como maligna) que afecta en su extensión a la base craneal anterior, obteniendo buenos resultados de resección y baja morbilidad. Aún así, en la actualidad tenemos un 24-56\% de complicaciones post-quirúrgicas según las series más recientes ${ }^{9,11,21,24}$, entre las cuales destacan la fístula de LCR y la meningitis, que son las más frecuentes y las que comportan mayor morbimortalidad.

\section{Complicaciones neurológicas}

La complicación neurológica más frecuente de nuestra serie fue el hematoma epidural agudo, que se presentó en 4 casos $(9.7 \%)$, siendo sólo sintomático en un paciente en el cual fue necesaria la re-intervención. En la literatura revisada se aprecia una incidencia de $2.3-6 \%{ }^{8,16}$ y suele presentarse en la fase precoz del post-operatorio, empeorando el pronóstico final en la mayoría de los casos ${ }^{8}$. En nuestra serie, a pesar de aparecer una incidencia algo superior a la de la literatura, los 4 pacientes evolucionaron favorablemente.

La meningitis fue diagnosticada en el 7.3\% de nuestros pacientes. La discusión de esta complicación la desarrollaremos más adelante junto a las fístulas de LCR, para su mejor comprensión.

El neumoencéfalo aparece en el post-operatorio precoz y constituye una complicación potencialmente fatal, cuando es a tensión $4,{ }^{28}$. El colgajo pediculado pericraneal puede actuar como un mecanismo valvular, atrapando el aire en el espacio epidural, y el drenaje lumbar puede facilitar su aparición y desarrollo. Cuando es sintomático puede requerir la aspiración percutánea ${ }^{28}$. En nuestra serie se detectaron 2 casos de neumoencéfalo (4.9\%), los dos asintomáticos fueron detectados en la TC craneal de control post-operatoria. El hecho de no utilizar drenaje lumbar profiláctico puede haber colaborado en la escasa relevancia de esta complicación en nuestra serie.

\section{Complicaciones locales}

La formación de espacios muertos, presencia de tejido necrótico y la RDT previa son los factores más importantes que predisponen a la infección de la herida quirúrgica ${ }^{18,19}$ Esta fue la complicación más frecuente, con una incidencia del 24.3\% (10 casos). En 3 casos fue necesario el desbridamiento quirúrgico, además del tratamiento antibiótico. Siete pacientes habían recibido RDT previa. Tres de estos siete pacientes (42.8\%), presentaron infección de herida quirúrgica, lo que hace suponer que la RDT puede jugar un papel importante en la desvitalización y predisposición a la infección del tejido.

La fístula de LCR constituye la complicación más frecuente (8.2-24\%) en la mayoría de publicaciones ${ }^{8,12,16}$; en nuestra serie fue la segunda complicación local más frecuente $(12.1 \%)$. Es una complicación potencialmente grave, que puede complicarse con meningitis (la causa de muerte en la literatura y $2^{\mathrm{a}}$ en nuestra serie); por ese motivo es esencial su correcto diagnóstico y tratamiento. Un gran defecto dural, el cierre dural no hermético, presencia de celdillas etmoidales o el seno esfenoidal abierto y la hidrocefalia ${ }^{28}$ son los factores predisponentes más importantes para el desarrollo de esta complicación ${ }^{7,20}$. En el $25 \%$ de los pacientes en que existía infiltración dural tumoral (3 de 12) apareció fístula de LCR, lo que parece relacionar la alteración tumoral de la barrera meníngea con la aparición de esta complicación (Tabla 3). El tratamiento de la fístula de LCR consiste en reposo en cama, colocar la cabecera 
del enfermo a $0^{\circ}$ y drenaje lumbar. En nuestra serie este tratamiento resolvió el 100\% de las fístulas. Si persiste será necesaria la reparación quirúrgica ${ }^{9}$. Algunos autores ${ }^{9,20,21}$ utilizan el drenaje lumbar tras la inducción anestésica para, además de facilitar el abordaje quirúrgico, prevenir la aparición de fístula de LCR. Existen controversias en cuanto a su uso preventivo, ya que el drenaje lumbar no es inocuo y puede tener complicaciones, tales como el neumoencéfalo. En nuestra serie aparecieron un $13 \%$ de fístulas de LCR sin utilizar drenaje lumbar preventivo, resultados similares a otras series $(8.2-20 \%)$ en las que sí se utiliza drenaje lumbar tras la inducción anestésica ${ }^{9,20,21}$, motivo por el cual creemos que el drenaje lumbar sólo debe utilizarse en aquellos casos que exista fístula de LCR o gran probabilidad de que esta ocurra. El cierre dural hermético y la utilización del colgajo pericraneal para tapizar la base craneal son las maniobras más efectivas en la prevención de la fístula de LCR. Probablemente, la utilización de un colgajo galealpericraneal (no utilizado en nuestra serie) podía disminuir aún más la aparición de esta complicación, al disponer de una mayor superficie para restablecer la barrera frontobasal y aerodigestiva.

La meningitis es una complicación que comporta alta morbi-mortalidad y que tiene como factor predisponente más importante la previa presencia de fístula de LCR. En nuestra serie diagnosticamos un $7.3 \%$ (3 casos) de meningitis, todos ellos tras la existencia de fístula de LCR, cifras comparables al $5-7.7 \%$ que se recoge en la literatura ${ }^{8,9,12,16,26}$. En el $16.6 \%$ de los casos en los que existía infiltración dural tumoral (2 de 12) apareció meningitis, lo que también parece relacionar la alteración tumoral de la barrera meníngea con esta complicación (Tabla 3). En nuestra serie, sólo uno de los tres casos evolucionó hacia el fallecimiento; en el resto, la evolución fue favorable, a diferencia de la elevada mortalidad (87.5\%) que presentan otros autores como Dias?. Existen otros factores que facilitan el desarrollo de esta complicación tales como el elevado tiempo quirúrgico, la contaminación multimicrobiana asociada a grandes tumores cutáneos ulcerados ${ }^{9}$, la radioterapia previa (>60 Gy) y la lobectomía frontal. En dos de nuestros casos (66\%) la cirugía se prolongó más de 6 horas. Es conveniente utilizar una correcta profilaxis antibiótica, así como el colgajo pediculado pericraneal como soporte para los lóbulos frontales, aislando así los dos compartimentos (intracraneal y cavidad aerodigestiva). Su utilización ha demostrado disminuir el riesgo de fístula de LCR, de infección y de encefalocele ${ }^{28}$.

El fallo del colgajo libre microvascularizado y colgajo local pediculado apareció en 2 ocasiones en el primero y 4 en el segundo (Tabla 3). Esta complicación aparece en la fase precoz y sus factores predisponentes son la infección de la herida quirúrgica y la hipotensión prolongada. La RDT juega un papel fundamental en la viabilidad de estos colgajos. En pacientes en que se haya realizado RDT previa, sería preferible utilizar colgajos libres de tejidos no irradiados para la reconstrucción.

La diplopia post-quirúrgica suele ser transitoria y generalmente se produce por pérdida de estructuras de soporte de la órbita, por lo que es necesario (en pacientes en los que no se ha realizado exanteración orbitaria) reconstruir la pared medial de la órbita ${ }^{2}$. Puede ser de utilidad un injerto óseo o un colgajo libre microvascularizado ${ }^{19}$. En nuestra serie se presentó en el 7.3\% (3 casos), a pesar de realizar una reconstrucción orbitaria y del ligamento cantal satisfactoria.

Presentamos un sólo caso de osteomielitis del colgajo óseo frontal $(2.4 \%)$, asociada a infección de la herida, en la que fue necesaria la extirpación del colgajo óseo y antibioterapia. Solero ${ }^{28}$ recomienda utilizar una craneotomía pequeña para disminuir la incidencia de osteomielitis del colgajo óseo, aunque en general, la mayoría de autores utilizan una craneotomía bifrontal amplia, sin por ello presentar una mayor incidencia de osteomielitis frontal ${ }^{3,5}$.

\section{Complicaciones sistémicas}

Las complicaciones sistémicas más relevantes de nuestra serie fueron las infecciones respiratorias (15.4\%), predominantemente en pacientes mayores 65 años y con un tiempo quirúrgico prolongado (4 de 6). El shock séptico apareció en 2 casos (4.8\%) y fue la causa de muerte en los dos pacientes.

\section{Conclusiones}

A pesar de los avances que han revolucionado la CCF y la han convertido en el principal tratamiento de esta patología, continúa asociándose a una elevada morbi/mortalidad, en general de tipo infeccioso.

El colgajo pericraneal ha demostrado ser muy eficaz para impermeabilizar el contenido intracraneal, disminuyendo la incidencia de fístulas de LCR e infecciones. Probablemente la utilización de un colgajo galeal-pericraneal mejoraría los resultados finales, disminuyendo aún más la aparición de estas complicaciones.

El drenaje lumbar debe utilizarse ante la presencia de fístula de LCR, no de forma preventiva, ya que puede aumentar la incidencia de neumoencéfalo.

El meticuloso cierre de todos los planos quirúrgicos, la ausencia de espacios muertos y de tejidos desvitalizados son los principales factores que evitarán la aparición de las complicaciones más frecuentes y peligrosas.

\section{Bibliografía}

1. Brasnu, D., Laccourreye, O., Menard, M., Devaux, B., 
Roux, F.X.: Transfacial approaches of cancers of the ethmoid. Neurochirurgie 1997; 43: 88-91.

2. Bebear, J.P., Darrouzet, V., Stoll, D.: Surgery of the anterior skull base: Total ethmoidectomy for malignant ethmoidal tumors. Isr J Med Sci 1992; 28: 169-172.

3. Bilsky, M.H., Kraus, D.H., Strong, E.W. et al.: Extended anterior craniofacial resection for intracranial extension of malignant tumors. Am J Surg 1997; 174: 565-568

4. Boyle, J.O., Shah, K.C., Shah, J.P..: Craniofacial resection for malignant neoplasms of the skull base: An overview. J Srg Oncol 1998; 69: 275-284.

5. Cantù, G., Solero, C.L., Mariani, L., Salvatori, P., et al.: Anterior craniofacial resection for malignant ethmoid tumors. A series of 91 patients. Head Neck 1999; 21:185-191.

6. Cophignon, J., George, B., Marchac, D., Roux, F.: Enlarged transbasal approach by mobilization of the medial fronto-orbital ridge. Neurochirurgie 1983; 29: 407-410.

7. Dandy, W.E.: Orbital tumors. Results following the transcranial operative attack. New York: Oskar Priest 1941: 1154.

8. Deschler, D.G., Gutin, P.H., Mamelak, A.N., et al.: Complications of anterior skull base surgery. Skull base surg 1996; 6: 113-118.

9. Dias, F.L., Sa, G.M., Kligernan, J, et al.: Complication of craniofacial resection. Head Neck 1999; 21: 12-20.

10. George, B., Salvan, D., Luboinski, B., Boissonnet, H., Lot G.: Malignant tumors of the ethmoid sinuses. A homogeneous series of 41 cases operated on by mixed approaches. Neurochirurgie 1997; 43: 121-124.

11. Gutiérrez Díaz, R., Sánchez Aniceto, G., Romance, A., Lobato, R., Cabrera, A., Alday, R.: Resección craneofacial anterior. Neurocirugía 1999; 10: 277-283.

12. Irish, I.C., Gulane, P.J., Gentili, F., et al.: Tumors of the skull base: outcome and survival of analysis of 77 cases. Head Neck 1994; 16: 3-10.

13. Kelly, M.B.H., Waterhouse, D., Slade, E., et al.: A 5year review of 71 consecutive anterior skull base tumors. Br J Plastic Surg 1999; 53: 184-190.

14. Ketcham, A.S., Van Buren, J.M.: Tumors of paranasal sinuses: a therapeutic challenge. Am J Surg 1985; 150: 406418.

15. Ketcham, A.S., Wilkins, R.H., Van Buren, J.M., Smith, R.R.: A combined intracranial facial approach to the paranasal sinuses. Am J Surg 1963; 106: 698-701.

16. Kraus, D.H., Shah, J.P., Arbit, E.: Complications of craniofacial resection for tumors involving the anterior skull base. Head Neck 1994; 16: 307-312.

17. Lesoin, F., Pellerin, P., Villette, L., Autricque, A., Jomin, M.: Mobilization of the median fronto-orbital band using a monobloc flap. Neurochirurgie 1986; 32: 161-163.
18. Lobato, R.D., Romance, A., Sánchez Aniceto, G., Cabrera, A., Rivero, B., Campollo, J.: La translocación facial ampliada en la resección de tumores de la base craneal. Nuestra experiencia con tres casos. Neurocirugía 1997; 8: 302-310.

19. Miller, M.J., Schusterman, M.A., Reece, G.P., et al: Microvascular craniofacial reconstruction in cancer patients. Ann Surg Oncol 1995; 2: 145-150.

20. Nibu Ken-ichi, Sasaki, Tomio, Kawahara, Nobutaka, et al: Complications of craniofacial surgery for tumors involving the anterior cranial base. Clinical study. Neurosurgery 1998; 42: 455-462.

21. Patel, S.G., Singh, B., Polluri, A., Bridger, P.G. et al.: Craniofacial surgery for malignant skull base tumors. Report of an international Collaborative study. Cancer 2003; 98: 1179-1187.

22. Roux, F.X., Brasnu, D., Menard, D., Schwaab, G. et al.: Combined approach to malignant tumors of the ethmoid and other paranasal sinuses: Principles and results. Ann Otolaryngol Chir Cervicofac 1991; 108: 292-297.

23. Roux, F.X., Devaux, B., Nataf, F., Pages, J.C., Laccourreye, O, et al:: Malignant tumors of the ethmoid region. Neurosurgical techniques. Neurochirurgie 1997; 43: 92-99.

24. Sánchez Aniceto, G., Romance García, A., Gutiérrez Díaz, R., Montalvo Moreno, J., Díez Lobato, R., Cabrera, A.: Métodos reconstructivos en cirugía tumoral de base de cráneo. Neurocirugía 1999; 10: 108-118.

25. Schramm, V.L., Myers, E.N., Maroon, I.C.: Anterior skull base surgery for benign and malignant disease. Laryngoscope 1979; 89: 1077-1091.

26. Schwaber, M.K., Nettervile, J.L., Conigliu, J.U.: Complications of skull base surgery. Ear Nose Throat J 1991; 70: 648-660.

27. Shah, J.P., Galicih, J.H.: Craniofacial resection for malignant tumors of the ethmoid and anterior skull base. Arch Otolayngol 1976; 108: 514-517.

28. Solero, C.L., Dimeco, F., Sampath, P., et al.: Combined anterior craniofacial resection for tumors involving the cribiform plate: early postoperative complications and thechnical considerations. Neurosurgery 2000; 47: 1296- 1304.

29. Sundarosan, N., Shah, J.P.: Craniofacial resection for anterior skull base tumors. Head Neck Surg 1988; 10: 219224.

Torres, A.; Acebes, J.J.; López, L.; Marnov, A.; Viñals, J.; Serra, J.M.; Mañosa, M.; Monner, A.: Complicaciones de la cirugía craneofacial en tumores de base craneal anterior. Neurocirugía 2005; 16:492-498.

Correspondencia postal: Alberto Torres Díaz. C/ Consejo de Ciento $n^{\circ} 1817^{\circ} 1^{\mathrm{a}} .08015$ Barcelona. 